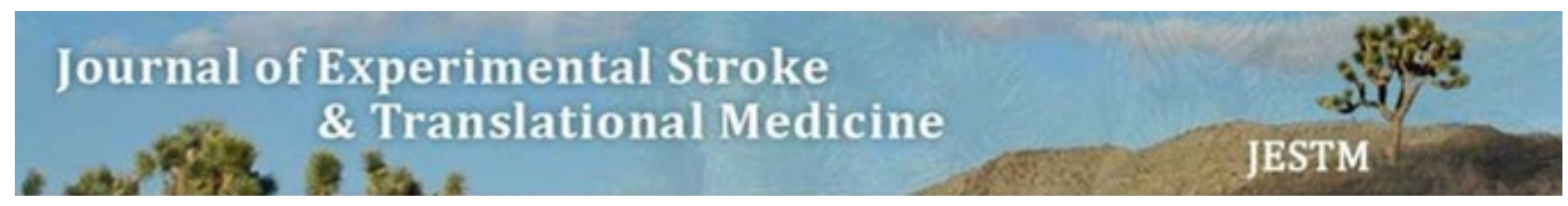

\title{
MacGreen mice: a novel tool to investigate inflammation following experimental stroke
}

\author{
September 29, 2015 · Volume $8 \cdot$ Original Research
}

Siyi Chen ${ }^{1,2}$, Laura Bennet ${ }^{3}$, Ailsa L. McGregor ${ }^{1,2}$

1School of Pharmacy, Faculty of Medical and Health Sciences, University of Auckland, Private Bag 92019, Auckland, New Zealand ${ }^{2}$ Center for Brain Research Faculty of Medical and Health Sciences, University of Auckland, Private Bag 92019, Auckland, New Zealand ${ }^{3}$ Department of Physiology, Faculty of Medical and Health Sciences, University of Auckland, Private Bag 92019, Auckland, New Zealand

Article Citation: Chen S, Bennet L, McGregor AL. MacGreen mice: a novel tool to investigate inflammation following experimental stroke: Mac Green mice and stroke. J Exp Stroke Transl Med 2015 Nov 1, Volume 8. 1-9. Online access at

Correspondence should be sent to: Dr Ailsa McGregor, School of Pharmacy and Centre for Brain Research, Faculty of Medical and Health Sciences, University of Auckland, Private Bag 92019, Auckland, New Zealand, Phone: +64 9373 1882, Email: ailsa.mcgregor@ auckland.ac.nz

\section{Abstract}

Background and Purpose: CSF-1R-EGFP ('MacGreen') transgenic mice carry an enhanced green fluorescent protein (EGFP) reporter gene in monocyte and macrophage populations. MacGreen mice have been used as a model system to investigate macrophage development and function. The current study investigated brain inflammation following experimental stroke.

Methods: MacGreen mice were subjected to transient middle cerebral artery occlusion and neurological deficit and ischemic damage compared to C57BI/6j mice. EGFP expression was also characterized at $24 \mathrm{~h}$ and 7 and 35 days post stroke in MacGreen mice.

Results: MacGreen mice show a comparable response to ischemia and progression of damage to C57BI/6j mice. Inflammation was related to stroke severity at both acute (24h) time points and several weeks ( 35 days) after experimental stroke. The increased EGFP signal in the ipsilateral hemisphere post stroke was attributed to both increased cell density and increased cell size. EGFP positive cells co-labelled with the microglia/macrophage marker Iba1 and changes in the morphology of these cells from $24 \mathrm{~h}$ to 7 and 35 days suggest temporal changes in the function of microglia/macrophages within ischemic regions.

Conclusion: MacGreen provide a clinically relevant platform in which to investigate the role of inflammation in the pathogenesis and recovery from stroke.

Key Words: Inflammation, Ischemic stroke, MacGreen Mice, Macrophage, MCAo, Microglia.

\section{Introduction}

Inflammation plays an important role in the pathogenesis of stroke and ischemic brain injury. Focal cerebral ischemia induces an acute response within the brain characterized by increased microglial and astrocyte activity, increased production of cytokines, chemokines, adhesion molecules and metalloproteinases and the infiltration of monocytes and leucocytes from the systemic circulation into injured brain regions(Jin R et al., 2010, Kriz J., 2006). These mediators display a specific temporal expression. When well-controlled, the inflammatory process is normally beneficial (Nathan C, 2002); Indeed, several traditionally 'pro-inflammatory' cytokines and chemokines including TNF $\alpha$ and stromal cell derived factor facilitate long-term behavioral recovery(Stahel PF et al., 2000, Scherbel U et al., 1999, Kriz J, Lalancette-Hebert M, 2009). In contrast to this, the overproduction of 'pro-inflammatory' cytokines certainly contributes to stroke pathophysiology(ladecola C, Anrather J, 2011).

The time-course of recruitment of inflammatory cells following cerebral ischemia has previously been investigated using techniques such as flow cytometry, RT-PCR and cytokine immunoassays(Stevens SL et al. , 2002, Matsumoto H et al. , 2008, Ormstad H et al. , 2011, Gelderblom M et al. , 2009). These studies typically require multiple cohorts of animals to satisfy tissue processing requirements and it is difficult to assess both the phenotype and anatomical distribution of inflammatory cells that have been activated in response to an ischemic injury in the same animals. The development of GFP expressing bone marrow chimeric mice has provided additional information on the origin of these cells and the mechanisms of recruitment. Following a mild ischemic insult, endogenous microglial activation occurs rapidly and precedes significant recruitment of blood-borne macrophages(Besselmann M et al. , 2003, Schilling M et al. 2005). Both MCP-1 and CCR2 are necessary for the recruitment of peripheral macrophages but not resident microglia(Strecker JK et al. , 2009, Ringelstein EB et al. , 2009). In contrast to this Tanaka et al(Tanaka R et al. , 2003) demonstrated an absence of microglia but a similar time-course of recruitment of blood-borne macrophages into the ischemic core 7 days following permanent MCAo. The heterogeneity of the inflammatory response in these studies suggests that the severity and location of ischemic damage has an impact on inflammatory cell recruitment. The application of irradiation chimera models to cerebral ischemia studies is further limited by reports of altered blood brain barrier integrity, cell death and microglial activation(Li Y-Q et al. , 2004, Monje ML et al. , 2002, Davoust N et al. , 2008, Mildner A et al. , 2007, Wirenfeldt $\mathrm{M}$ et al. , 2007). The relationship between ischemic damage and inflammatory response in the weeks following ischemia has yet to be investigated in a transgenic GFP reporter line. CSF1-EGFP ('MacGreen') mice carry an enhanced green fluorescent protein (EGFP) reporter gene which is under control of the colony stimulating factor-1 (CSF-1) receptor promoter. CSF-1R is encoded by the 
c-fms proto-oncogene which is expressed selectively in macrophage and trophoblast cells. This model system has been used to isolate macrophages from embryonic mice and investigate macrophage function during development(Rae F et al. , 2007, Sasmono RT et al. , 2003, Sasmono RT et al. , 2007). EGFP expression is restricted to macrophage populations in bone marrow, blood and all adult tissues EGFP is also expressed in microglia and in brain macrophages associated with the vasculature and we propose that EGFP expression will be up-regulated following an ischemic insult. Investigating long-term inflammation in this clinically relevant model may improve our understanding of the pathogenesis and recovery from stroke.

The aim of this study was therefore to investigate the response of MacGreen mice to different periods of middle cerebral artery occlusion compared to a standard mouse model used for focal cerebral ischemia studies and to investigate the relationship between structural lesion and EGFP expression at 24 hours and 7 and 35 days post stroke.

\section{Material and Methods}

\section{Ethical Statement}

All animal work was carried out in accordance with the Animal Welfare act 1999 and was pre-approved by the University of Auckland Animal Ethics committee (Approval number: R842). All surgery was performed under isoflurane anesthesia and all animals received pre and post-operative analgesia to minimize suffering.

\section{Animals}

Founder CSF-1R-EGFP (MacGreen) mice were gifted by the Queensland Brain Institute, University of Queensland, Australia. Generation of the strain has been described elsewhere(Sasmono RT et al., 2003). The MacGreen colony was maintained as homozygote and all offspring were positive for the transgene. The ongoing presence of the transgene within the colony was confirmed every 2 months from tail biopsies using standard PCR methods. Tail tipping was performed under isoflurane anesthesia. DNA extraction from tail biopsies was performed using REDExtract-N-Amp Tissue PCR kit (Sigma-Aldrich, USA) using forward 5'-CTGGTCGAGCTGGACGGCGACG-3' and reverse 5'-CACGAACTCCAGCAGGACCATG-3' primers, producing a 650bp product.

Age matched male C57BI/6j mice obtained from stock colonies within the University of Auckland animal facility were used as controls.

All animals were housed in single sex cage and supplied with normal rodent chow and water available ad libitum. Housing Bio-bubbles were maintained at $20^{\circ} \mathrm{C}$ with 12 hour day and night cycle.

\section{The Cerebral ischemia}

Male C57BI/6j and CSF-1R-EGFP 'MacGreen' mice (25-30g, approximately 10-12 weeks) were assigned to experimental groups using a randomization table generated by Excel. Core temperature was maintained at $36.5^{\circ} \mathrm{C} \pm 0.5^{\circ} \mathrm{C}$ throughout the course of the surgery by means of a rectal probe connected to a homeothermic heating blanket (Harvard Apparatus, U.S.A.)

Monofilament occlusion of the middle cerebral artery (MCA) was performed in adult male transgenic mice and C57BI/6j controls (25-30g, approximately 10-12 weeks) as previously described (Kerr et al. , 2004). Briefly, the right common carotid (CCA), external carotid (ECA) and internal carotid (ICA) arteries and their branches were exposed through a midline cervical incision. A 6-0 silk suture was tied around the CCA proximal to the bifurcation of the ECA and ICA and a second suture tied around the ECA distal to the superior thyroid artery (STA). The STA and occipital artery (OA) were occluded by electrocoagulation. An 8-0 silicone-coated monofilament (diameter $200 \mu \mathrm{m}$ ) was introduced into the CCA and advanced $10 \mathrm{~mm}$ distal to the carotid bifurcation, occluding the origin of the MCA

After surgery, animals were allowed to recover in a humidified and temperature-controlled incubator for at least 2 hours until they were moving freely. Animals were then returned to their home cages.

C57BI/6j and MacGreen mice were subjected to MCA occlusion for 30 ( $n=4$ per strain), 45 (n=4-6 per strain) or 60 minutes ( $n=3-4$ per strain) and killed at 24 hours. A further group of mice were subjected to 45 min MCAo were killed at 35 days ( $n=5$ per strain) to investigate lesion progression. A second cohort of MacGreen mice were subjected to 45 min MCA and killed at $7(n=3)$ and 35 ( $n=3)$ days to investigate spatiotemporal EGFP expression.

\section{Statistical analysis}

Values presented in this study are mean +/- s.e.m. The randomization code was broken after all data was acquired to allow allocation to experimental groups. All statistical analyses were carried out using SPSS (PASW Statistics 18, 2009). Two-way Analysis Of Variance (ANOVA) was used to determine whether statistically significant differences were present in neurological score and infarct volumes between MacGreen and C57BI/6j mice. Significant differences in tissue loss were assessed using an unpaired Student t-test.

Comparison of EGFP expression in MacGreen mice at 24 hours and 35 days was conducted using one-way ANOVA. The relationship between lesion volume and EGFP expression was determined using non-linear regression analysis. Statistical significance was established at $p<0.05$

\section{Results}

\section{Ischemic damage at 24 hours}

To investigate whether C57BI/6j and MacGreen mice show differing sensitivity to ischemic brain damage mice were subjected to 30,45 and $60 \mathrm{~min}$ of middle cerebral artery occlusion (MCAo). Thionin staining revealed qualitatively different patterns of damage with increasing ischemic duration in both C57BI/6j and MacGreen mice (Figure 1A). A 30 min occlusion produced damage restricted to the striatum in both strains while the majority of those subjected to $45 \mathrm{~min}$ MCAo exhibited additional areas of ischemic damage in the overlying cortex. All animals subjected to $60 \mathrm{~min}$ MCAo had large infarcts with both cortical and striatal components, however C57BI/6j mice appeared to show a higher incidence of cortical damage (Figure 1A). Further analysis of the area of ischemic damage at 9 stereotaxic levels confirmed C57BI/6j mice subjected to $60 \mathrm{~min}$ MCAo displayed a larger area of cortical damage at Bregma $+1.5 \mathrm{~mm}$ compared to MacGreen mice $\left(F_{2,7}=4.74, p<0.01\right.$, Figure 1B-C). No damage was observed out with the vascular territory of the MCA in any of the animals investigated. 
The lesion progression in response to increasing duration of occlusion observed qualitatively was confirmed by two-way ANOVA which revealed a significant effect of ischemic duration $\left(F_{2.23}=17.64, p<0.01\right)$; Figure 1D), but no effect of strain, $\left(F_{223}=1.28, p=0.273\right)$. Post-hoc analysis revealed that mice subjected to $60 \mathrm{~min}$ MCAo had significantly larger lesion volumes compared to those subjected to $30 \mathrm{~min}$ and 45min MCAo $\left(F_{2.23}=17.64, p<0.01\right)$. Lesion volumes following 45min MCAo were not different from those following 30min MCAo ( $t=1.201$; $p=0.56)$.
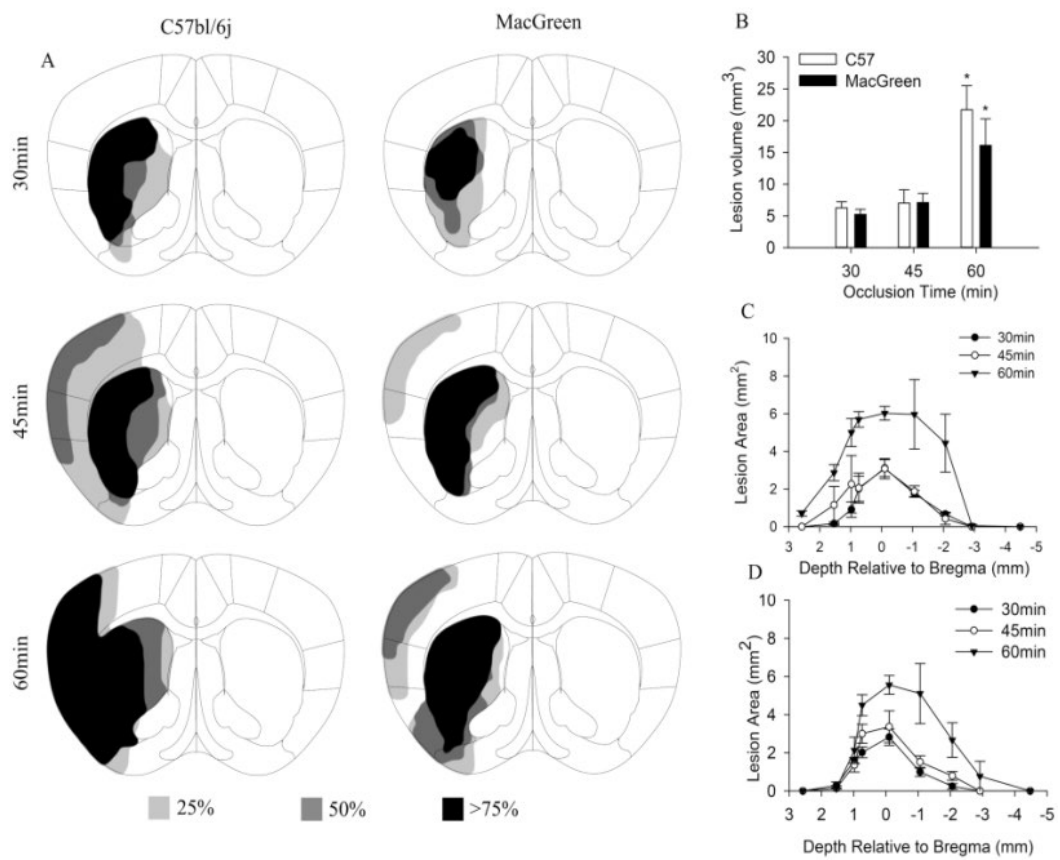

Figure 1.

(A) Incidence maps show patterns of ischemic damage at the level of the striatum (Bregma $+0.98 \mathrm{~mm}$ ) following 30,45 and $60 \mathrm{~min}$ of transient MCAo at 24 hours post stroke.

(B) Lesion volume at $24 \mathrm{~h}$ following transient (30, 45 or $60 \mathrm{~min})$ MCAo. Two-way ANOVA showed a significant effect of occlusion duration $\left(\mathrm{F}_{2,23}=17.64, p<0.01\right)$ but no effect of $\operatorname{strain}\left(\mathrm{F}_{1.23}=1.281 ; p=0.273\right)$. The area of ischemic damage at 9 stereotaxic levels through the rostro-caudal extent of the brain in $\mathrm{C} 57 \mathrm{BI} / 6 \mathrm{j}$

(C) and MacGreen mice

(D) Subjected to 30, 45 or 60 min MCAo. MacGreen mice displayed a smaller area of cortical damage than C56BI/6j mice at a single level $\left(B r e g m a+1.55 \mathrm{~mm}, \mathrm{~F}_{2,7}=4.74, p<0.01\right)$. Data are mean \pm s.e.m., $\mathrm{n}=4-6$ mice per group, ${ }^{*} p<0.05$.

\section{Neurological deficits}

All animals showed a decrease in neurological score post stroke and no significant differences were observed between MacGreen and C57BI/6j mice at 24 hours following mild $(30 \mathrm{~min})$, moderate $(45 \mathrm{~min})$ or severe $(60 \mathrm{~min})$ ischemia. Both strains subjected to a moderate insult displayed comparable neurological scores at 24 hours post stroke $(\mathrm{C} 57 \mathrm{Bl} / 6 \mathrm{j}=9.8 \pm 0.58$, MacGreen $=10.3 \pm 0.33$, Figure 2B). The survival rate out to 35 days following a moderate $(45 \mathrm{~min})$ insult was $100 \%$ for both strains. Both MacGreen and C57Bl/6j mice showed a partial recovery of neurological function and had comparable neurological scores at 35 days $(\mathrm{C} 57 \mathrm{BI} / 6 \mathrm{j}=11.8 \pm 0.49$, MacGreen $=12.33 \pm 0.33$, figure 2B). Two-way ANOVA confirmed a significant effect of day post stroke on neurological score $\left(F_{2,12}=22.80, p<0.01\right)$ but no effect of strain $\left(F_{2,12}=13.28, p=0.72\right)$.

\section{Ischemic damage at 35 days}

Morphological assessment of ischemic damage was also carried out 35 days post-stroke following 45 min MCAo in both strains of mice. In keeping with observations at 24 hours, thionin stained brain sections at 35 days showed ischemic damage in the lateral parts of the striatum and the overlying cortex of C57BI/6j mice (Figure 2A). In contrast, ischemic damage was restricted to the striatum in MacGreen mice subjected to $45 \mathrm{~min}$ MCAo. Despite this difference in infarct pattern, we observed no significant difference in lesion volumes between C57BI/6j $\left(5.74 \pm 1.43 \mathrm{~mm}^{3}\right)$ and MacGreen mice $\left(3.31 \pm 0.57 \mathrm{~mm}^{3}\right)$ at 35 days $\left(F_{18}=2.745, p=0.34\right.$, Figure $\left.2 \mathrm{C}\right)$. Quantitative histopathology further demonstrated no difference in atrophy in the ipsilateral hemisphere of C57BI/6j (18.6 $\pm 6.69 \%)$ and MacGreen mice $(6.6 \pm 6.80 \%$; $\mathrm{t}=2.374 ; p=0.055$; Figure 2D). 


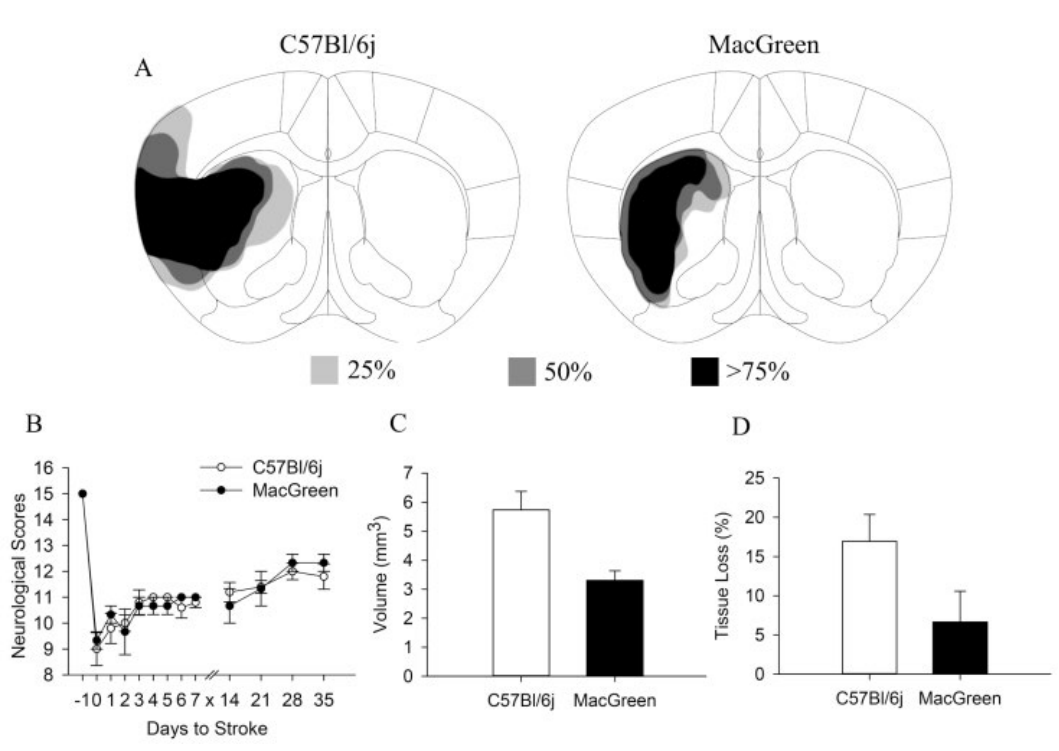

Figure 2.

(A) Incidence maps showing patterns of ischemic damage obtained in C57BI/6j and MacGreen mice at the level of the striatum (Bregma $+0.98 \mathrm{~mm}) 35$ days following a moderate $(45 \mathrm{~min})$ insult.

(B) MCAo produced an immediate decrease in neurological score in all animals $\left(F_{2,12}=22.80, p<0.01\right)$. Both strains of mice showed partial recovery of neurological function by 35 days post-stroke. No significant differences in neurological score were observed between MacGreen and C57BI/6j mice at any time point examined $\left(F_{1,103}=0.139 ; p=0.72\right)$.

(C) Despite C57BI/6j mice showing more cortical damage than MacGreen mice, 2-way ANOVA on total lesion volume at 35 days demonstrated no significant effect of strain $\left(\mathrm{F}_{1.8}=2.745 ; p=0.34\right)$.

(D) There was also no significant difference in the percentage tissue loss in the ipsilateral hemisphere between the 2 strains ( $\mathrm{t}=2.374 ; p=0.055)$. Data are mean \pm s.e.m., $\mathrm{n}=4-6$ mice per group.

\section{Increased EGFP expression in MacGreen mice 24 hours following cerebral ischemia}

Immunocytochemistry on brain sections from MacGreen mice subjected to sham stroke surgery showed a low basal level of EGFP expression (data not shown). In contrast, MacGreen mice subjected to 30, 45 and 60 min MCAo displayed increased EGFP expression in the ipsilateral hemisphere at 24 hours and no change in contralateral EGFP expression (Figure 3). Regions of increased EGFP expression broadly correlated with the pattern of ischemic damage. The volume of increased EGFP expression 24 hours following MCAo was significantly larger following a severe insult than after a mild or moderate insult $\left(F_{211}=9.64, p<0.01\right)$. In addition, the volume of increased EGFP expression in the ipsilateral hemisphere was significantly larger than the corresponding histological lesion volume $\left(F_{1,22}=32.8\right.$; $p<0.01$; Figure $4 \mathrm{~A}$ ). Regression analysis confirmed a significant exponential relationship between lesion volume and EGFP volume $\left(r^{2}=0.776 ; p<0.01\right)$, where mice with smaller histological lesions tended to have a smaller volumes of increased EGFP expression (Figure 4B). The calculated ratio of the volume of increased EGFP expression to the lesion volume assessed by thionin histology (EGFP/Thionin ratio) was approximately 2.8 and was consistent at different occlusion times $\left(F_{2,12}=0.006 ; p=0.939\right)$.

EGFP positive cells in the ipsilateral hemisphere had an amoeboid morphology with enlarged cell bodies and retracted processes. In contrast, EGFP positive cells in the contralateral hemisphere displayed a ramified morphology. Semi-quantitative analysis was performed to determine whether the increased EGFP expression in the ipsilateral hemisphere post-stroke resulted from an increased cell density or a change in cell morphology.

EGFP expression $/ \mu \mathrm{m}^{2}$ was increased $7-8$ fold in the ipsilateral striatum $24 \mathrm{~h}$ post stroke $\left(\mathrm{F}_{1.77}=6016.07 ; p<0.01\right.$; Figure $\left.4 \mathrm{C}\right)$. MCAo also induced a significant 1.5 fold increase in the number of EGFP positive cells in the ipsilateral hemisphere compared to corresponding regions within the contralateral hemisphere $\left(F_{1.25}=148.03 ; p<0.01\right.$, Figure 4D). The duration of occlusion did not influence EGFP signal intensity $\left(F_{2.77}=0.785 ; p=0.46\right)$ or cell counts or in either hemisphere $\left(F_{2.25}=1.142 ; p=0.339\right)$. 


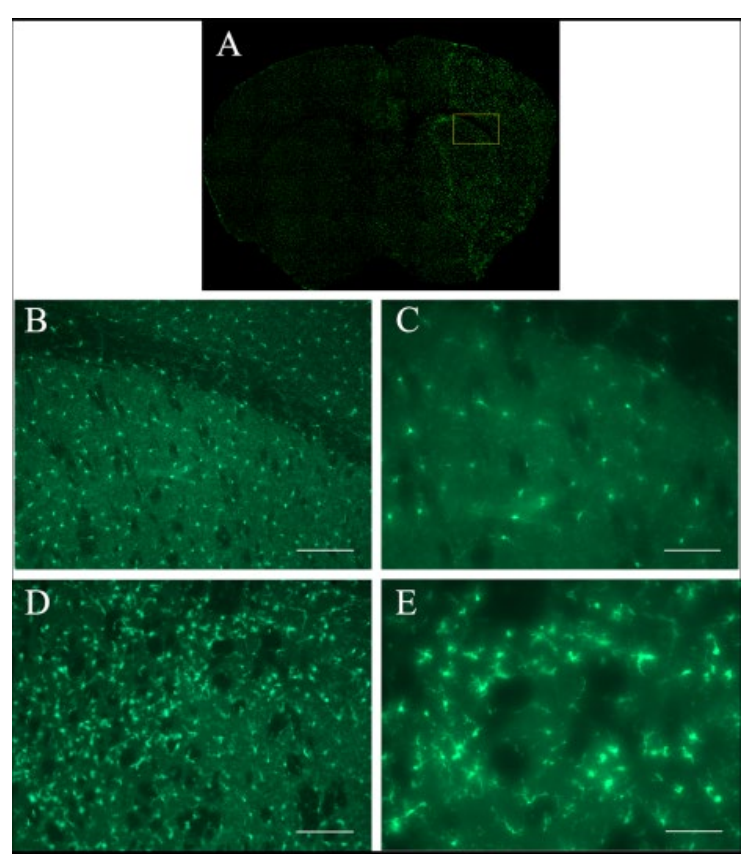

Figure 3.

Photomicrographs showing increased EGFP expression in MacGreen mice $24 \mathrm{~h}$ after a moderate ischemic insult (45 min). Images were taken at the level of the striatum (Bregma $+0.74 \mathrm{~mm}$ ). EGFP expression was upregulated in the striatum and sensory-motor cortex in the ipsilateral hemisphere (A). EGFP positive cells in the contralateral hemisphere displayed a ramified morphology $(B, C)$. MCAo increased the number of EGFP positive cells in the ipsilateral hemisphere. EGFP positive cells displayed an amoeboid morphology with enlarged cell bodies and retracted processes. Scale $=200 \mu \mathrm{m}(\mathrm{b}$, d), $100 \mu \mathrm{m}(\mathrm{c}, \mathrm{e})$.
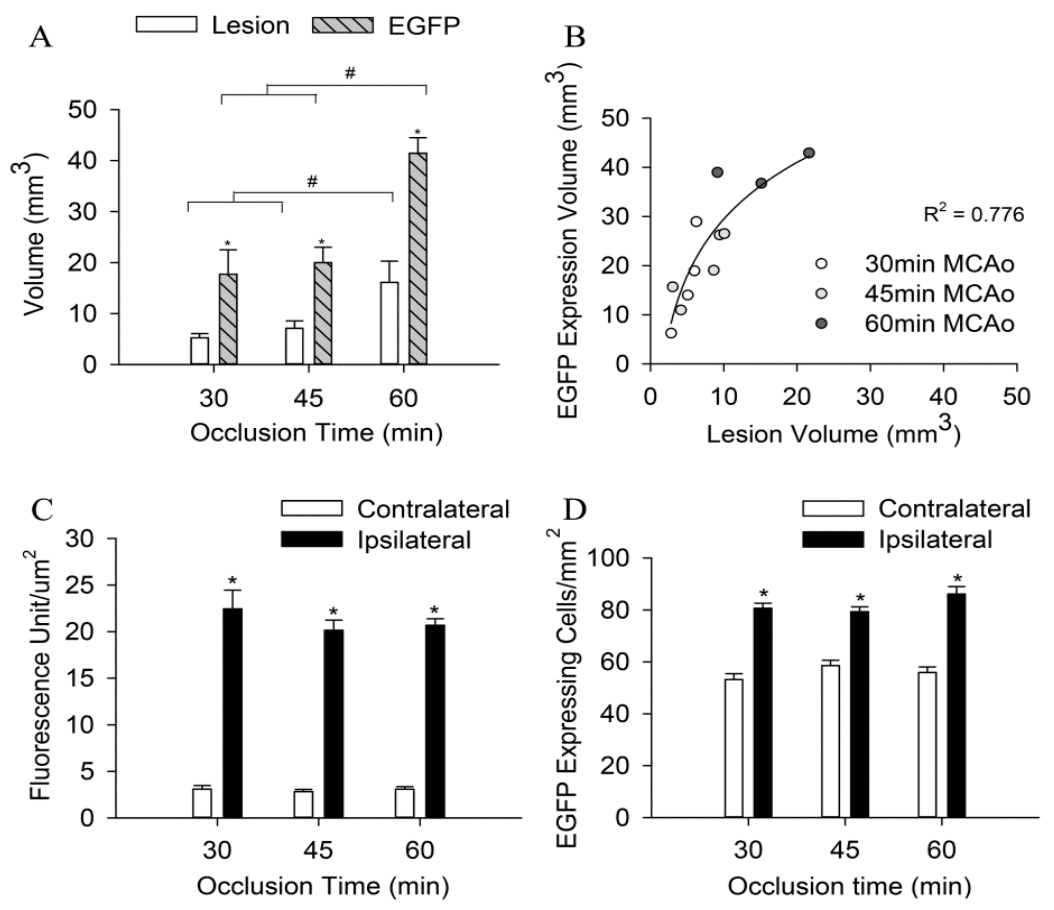

Figure 4.

Increasing the duration of occlusion in MacGreen mice increased the volume of both ischemic damage and EGFP expression 
(A) Animals subjected to 60 min MCAo had larger EGFP volumes and volumes of structural damage than following 30 or $45 \mathrm{~min}\left(\mathrm{~F}_{1,22}=32.8 ; p<0.01\right)$. The volume of EGFP expression in the ipsilateral hemisphere was significantly larger than the corresponding histological lesion volume at all severities of ischemic insult $\left(\mathrm{F}_{1,22}=32.8 ; p<0.01\right)$. Regression analysis confirmed a significant exponential relationship between lesion volume and EGFP volume $\left(r^{2}=0.776 ; p<0.01\right)$;

(B) Indirect quantification of the morphology of EGFP positive cells within the ipsilateral hemisphere demonstrated a 7-8 fold increase in EGFP expression $/ \mu \mathrm{m}^{2}$ in the ipsilateral striatum

(C) MCAo induced a significant increase in the estimated number of EGFP positive cells in the ipsilateral hemisphere compared to corresponding regions within the contralateral hemisphere $\left(\mathrm{F}_{1.77}=6016.07 ; p<0.01\right.$; $\left.\mathrm{D}\right)$. The duration of $\mathrm{MCA0}$ occlusion did not influence the intensity $\left(\mathrm{F}_{2,77}=0.785 ; p=0.46\right)$ or density of EGFP positive cells in either hemisphere $\left(\mathrm{F}_{2,25}=1.142 ; p=0.339\right)$. Data are mean \pm s.e.m., $n=4-6$ mice per group, ${ }^{*} p<0.05$ within time points, \#p<0.05 between time points.

\section{Increased EGFP expression at 7 and 35 Days in MacGreen mice}

Increased EGFP expression was also observed within the ipsilateral hemisphere 7 and 35 days following a $45 \mathrm{~min}$ MCAo (Figure $5 \mathrm{~A}$ ). In keeping with observations at $24 \mathrm{~h}$, the volume of increased EGFP expression in the ipsilateral hemisphere remained significantly larger than the corresponding histological lesion at both 7 and 35 days $\left(F_{21}=18.126 ; p<0.05\right)$. However, the volume of tissue showing increased EGFP expression at 35 days was decreased compared to $24 \mathrm{~h}\left(\mathrm{~F}_{1,21}=4.675 ; p<0.05\right)$, despite quantitative analysis confirming comparable structural lesions $\left(F_{121}=1.248 ; p=0.543\right)$. EGFP/thionin ratios were correspondingly decreased at 7 and 35 days post stroke $\left(24 \mathrm{~h}=3.09 \pm 0.54,7\right.$ days $=2.40 \pm 0.44,35$ days $=1.67 \pm 0.23 ; \mathrm{F}_{2,10}=2.154 ; p=0.179$, Figure $\left.5 \mathrm{~B}\right)$.

EGFP expression at 7 and 35 days post stroke appeared qualitatively different from that observed at 24 hours. EGFP positive cells were densely packed within a central 'core' and displayed a distinct amoeboid morphology with enlarged cell bodies and retracted processes (Figure $6 \mathrm{~A}, \mathrm{D}, \mathrm{G})$. EGFP signal intensity within the ipsilateral hemisphere increased significantly over time from 24 hours (20.1 \pm 4.2 units/ $\left.\mu \mathrm{m}^{2}\right)$ to $7\left(102.6 \pm 11.7\right.$ units $\left./ \mu \mathrm{m}^{2}\right)$ and 35 days $\left(128.8 \pm 18.3\right.$ units/ $\mu \mathrm{m}^{2} ; \mathrm{F}_{22}=288.809 ; p<0.01$, Figure $\left.5 \mathrm{C}\right)$. A similar pattern of increasing EGFP positive cell counts was also observed out to 35 days post-stroke $\left(24 \mathrm{hr}=95 \pm 9\right.$, 7 days $=1668 \pm 123,35$ days $=1920 \pm 90 ; F_{2,26}=1995.624$; $p<0.01$, Figure 5D).

A

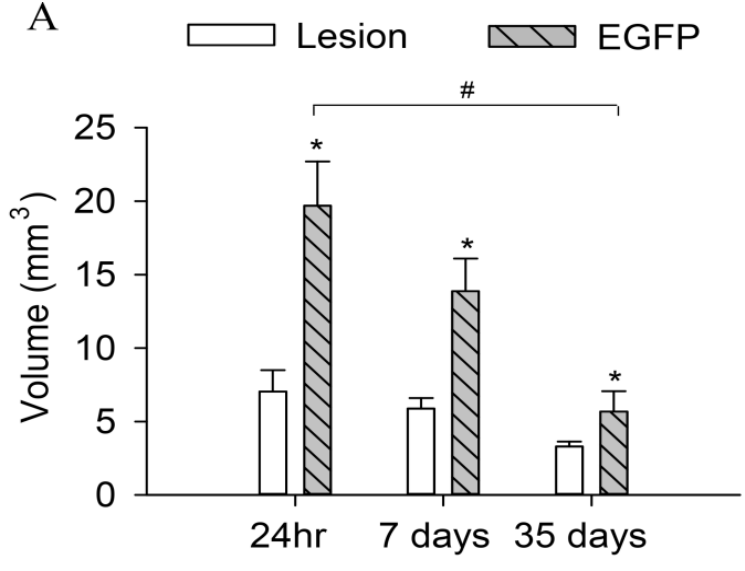

$\mathrm{C}$

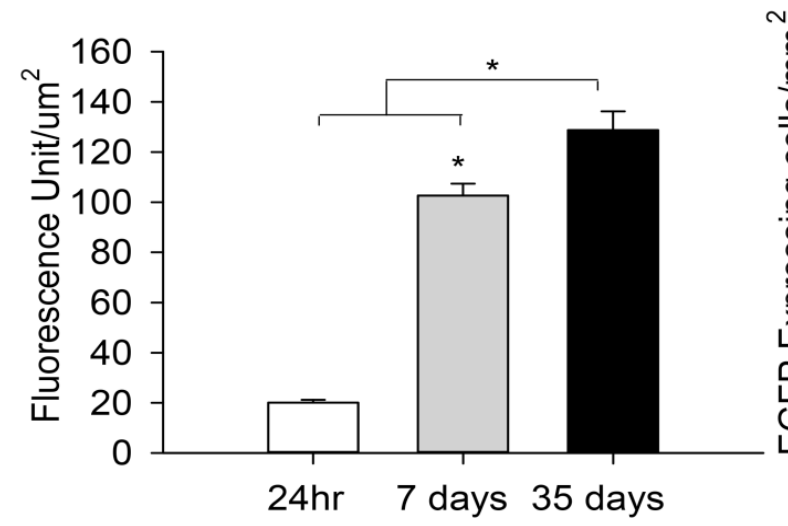

B
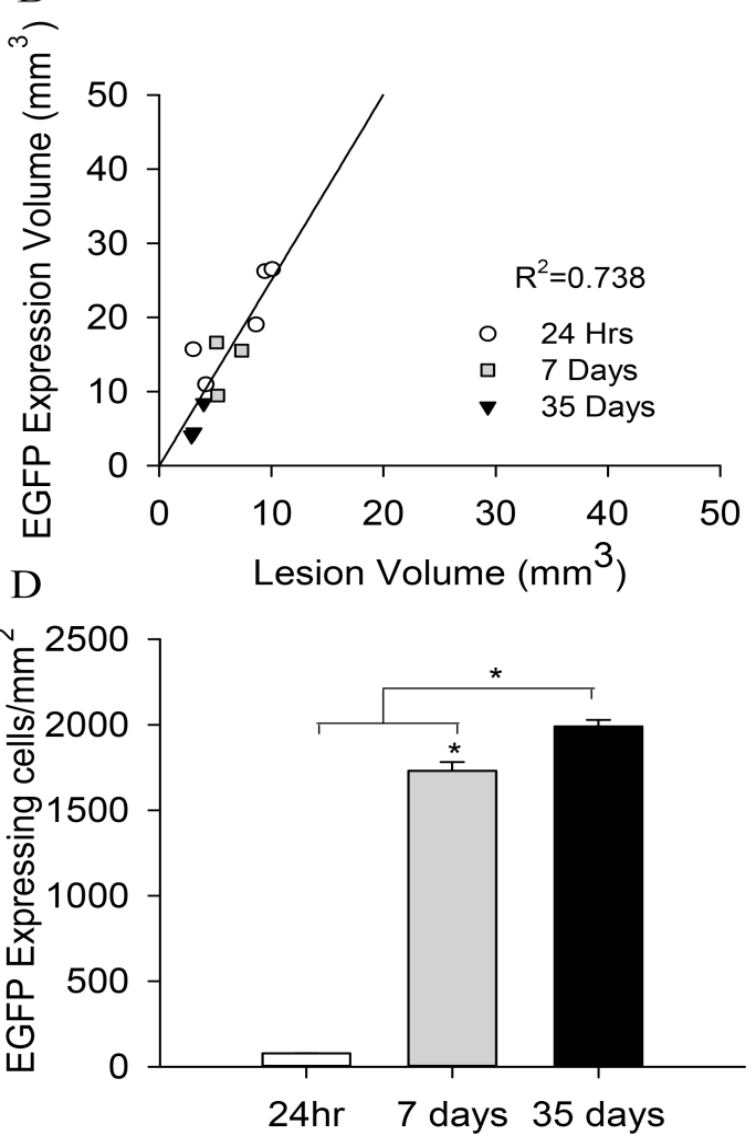

\section{Figure 5.}

EGFP expression remained elevated 7 and 35 days post stroke however the EGFP expression volume was decreased at 35 days compared to $24 \mathrm{~h}\left(\mathrm{~F}_{121}=4.675 ; p<0.05 ; \mathrm{A}\right)$. Regression analysis confirmed a significant exponential relationship between lesion volume and EGFP volume out to 35 days $\left(r^{2}=0.738 ; p<0.01 ; B\right)$. MCAo induced a significant timedependent increase in EGFP expression $/ \mu \mathrm{m}^{2}\left(\mathrm{~F}_{2}=288.809 ; p<0.01 ; \mathrm{C}\right)$ and an accumulation of EGFP positive cells in the ipsilateral striatum over time $\left(\mathrm{F}_{2,26}=1995.624 ; p<0.01\right.$; D). Data are mean \pm s.e.m., $\mathrm{n}=3-5$ mice per group, ${ }^{*} p<0.05$ within time points, $\# p<0.05$ between time points. 


\section{Phenotype of EGFP positive cells}

In order to determine the phenotype of the EGFP positive cells within the ischemic hemisphere, we performed double label immunohistochemistry with Iba1, a marker for microglia and monocyte lineage cells. Both EGFP and Iba1 positive cells displayed an irregular shape and enlarged cell bodies with retracted processes (Figure 6). Confocal microscopy confirmed that all of the EGFP positive cells co-localized with Iba1 in the ipsilateral (and contralateral) hemispheres at $24 \mathrm{~h}$. The majority of EGFP positive cells $(<99 \%)$ also coexpressed Iba1 and 7 and 35 days.
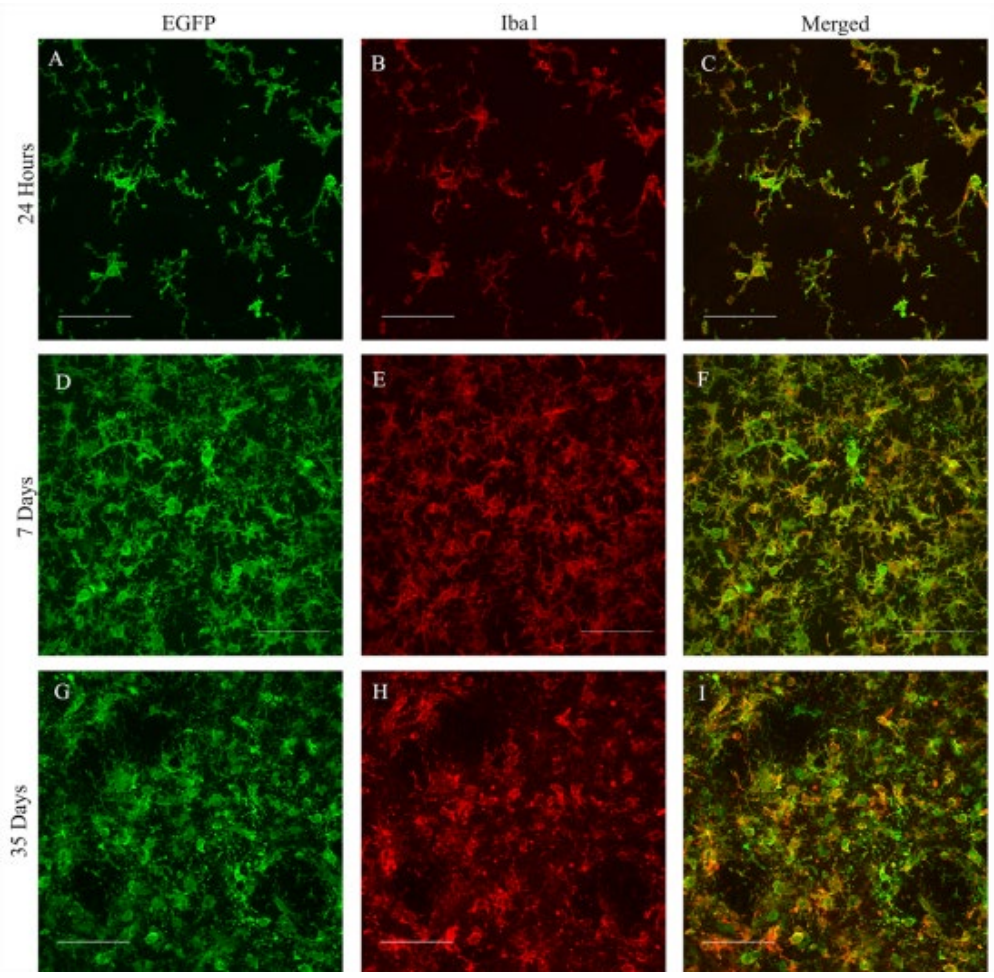

Figure 6.

Confocal images of EGFP (left panel) and Iba1 (middle panel) expression in MacGreen mice 24h (A-C) and 7 (D-F) and 35 days (G-I) following a 45 min MCA occlusion. Images were taken from the ipsilateral striatum at the level of Bregma $+0.74 \mathrm{~mm}$. Subtle differences in the distribution of the two markers are evident in cell bodies and processes, however all EGFP positive cells also co-express Iba1 (right panel). The density of EGFP positive cells was higher within the ipsilateral striatum at 7 and 35 days following a 45 min insult and cells show distinct morphological changes compared to $24 \mathrm{~h}$.

\section{Discussion}

MacGreen (Csf1r-EGFP) mice carry an enhanced green fluorescent protein (EGFP) gene downstream of the c-fms promoter. The c-fms gene encodes the receptor for macrophage colony stimulating factor (CSF-1) and is expressed selectively in macrophage and monocyte cell lineages (Sasmono RT et al., 2003). MacGreen mice provide a model system for the study of macrophage development and function in the periphery (Rae F et al., 2007, Sasmono RT et al., 2003, Sasmono RT et al., 2012, Jones CV et al., 2013) and we propose that that provide a unique platform in which to investigate inflammation in the ischemic brain. To our knowledge, the response of MacGreen EGFP reporter mice to experimental stroke has not previously been investigated.

We first characterized the susceptibility of MacGreen mice to different durations of MCAo in comparison to the commonly used C57BI/6j strain. Experimental stroke studies must demonstrate an acceptable rate of survival to ensure statistical power and to satisfy ethical requirements. All MacGreen and C57BI/6j mice subjected to MCAo (30, 45 and $60 \mathrm{~min})$ survived for 24 hours post-stroke and all of the mice subjected to $45 \mathrm{~min}$ MCAo survived to 35 days. We also did not observe any statistically significant differences in general welfare, indicated by weight loss, post-operatively. The neurological deficits observed 24 hours following MCAo were comparable and both strains demonstrate an increase in neurological score over time, suggesting a partial recovery of function which closely mirrors the clinical situation in human stroke patients (Hosp JA et al., 2011).

The patterns of ischemic damage produced by MCAo were consistent between the two strains and showed a typical progression with shorter occlusion times producing more discrete lesions. Further analysis of the rostro-caudal extent of ischemic damage revealed a significant decrease in lesion area at a single rostral stereotaxic level (Bregma +1.5) in MacGreen mice subjected to 60min MCAo compared to $\mathrm{C} 57 \mathrm{BI} / 6 \mathrm{j}$. This difference in the rostro-caudal extent of ischemic damage may result from differences in the cerebrovasculature of MCA for the two strains. The relationship between cerebrovascular territory and lesion volume has been well documented within the literature (Maeda K et al., 1999, Maeda K et al., 1999, Maeda K et al., 1998, Carmichael ST, 2005). For example, the resistance to ischemia observed in SV129 mice results from the small vascular territory supplied by the MCA in this strain (Maeda K et al., 1998). Regardless, the difference in the extent of ischemic damage at a single stereotaxic level was not sufficient to produce a significant difference in lesion 
volume between the two strains of mice. Therefore, we can conclude that both strains demonstrate comparable responses to different durations of MCA occlusion at 24 hours post-stroke.

We extended our investigation to examine the long term response to a moderate ischemic injury and found that MacGreen mice display a similar degree of ipsilateral hemispheric atrophy and comparable infarct volumes to C57BI/6j mice (Carmichael ST, 2005) days post-stroke despite a lower incidence of cortical damage in MacGreen mice. The functional consequences of decreased cortical injury in MacGreen mice were not investigated in this study however studies investigating cortical protection in traumatic brain injury have demonstrated reduced deficits in sensorimotor tasks such as tactile adhesive removal and locomotor placement tests (Goffus AM et al., 2010, Blaya MO et al., 2013).

EGFP expression in the MacGreen mouse is directed by the promotor for CSF-1R (c-fms) and is upregulated during differentiation and expansion of dendritic cells (MacDonald 2005). Since CSF-1R is reportedly upregulated by ischemic injury (Yu et al, 2008) we hypothesized that EGFP expression would also be upregulated in the MacGreen mouse brain following stroke. MCA occlusion induced an increase in EGFP expression in the ipsilateral hemisphere of MacGreen mice which was dependent on the severity of ischemia. While this pattern was comparable to the observed duration-dependent increases in the volume of structural damage post occlusion, EGFP volumes were typically 2.5 fold greater than the volume of infarct outlined thionin histology. This 'mismatch' between the structural lesion and volume of increased EGFP expression 24 hours post stroke suggests spill-over of inflammatory signals into the intact tissue surrounding areas of irreversible damage. We propose that increased EGFP expression out with areas of structural damage provides information on inflammation within the peri-infarct region while the EGFP: Lesion volume ratio, which was reduced at 7 and 35 days compared to 24 hours, may be a useful measure to assess the general inflammatory status within the brain.

The increase in EGFP expression in the ipsilateral hemisphere at 24h, 7 and 35 days results from both an increase in cell density and also an increase in EGFP expression per unit area which indirectly corresponds to altered cell morphology. EGFP positive cells display processes and enlarged cell bodies and these changes in morphological appearance an important indicator of functional activation (Perego C et al., 2011, Yenari MA et al., 2010, Ransohoff RM et al. 2009).

All EGFP cells at $24 \mathrm{~h}$ co-localized with lba1 confirming these cells are microglia/monocyte lineage. While the origin of these cells remains unknown, (Schilling et al., 2003) report that in an irradiation chimeric model the majority of macrophages in the infarct area are derived from local microglia, and that local defense mechanisms predominate over hematogenous infiltration at earlier time points post stroke (Gelderblom M et al., 2009, Besselmann M et al., 2003)

While the absolute volume of EGFP expression decreased over time, we observed continued accumulation of EGFP positive cells in the ipsilateral hemisphere from 7 to 35 days post stroke demonstrating that the inflammatory processes continues for at least 5 weeks after stroke(Schroeter M, 1994, Gomes-Leal W et al., 2012). EGFP positive cells in the striatum at 7 and 35 days appeared more densely packed and had rounder cell bodies with shorter processes than in comparable regions at 24 hours which may reflect a change in microglial/ macrophage/monocyte (M/M) function (Perego C et al., 2011). A very recent paper by Ritzel et al which allowed discrimination between cell populations using the phenotypic marker CD45 showed a decrease in microglial activity and an increased contribution to phagocytosis from infiltrating monocytes 3-7 days after a severe ischaemic insult (Ritzel RM et al., 2015)These monocyte-derived macrophages appear to be essential to debris clearance and functional recovery. Since there appears to be little contribution to phagocytosis from monocytederived macrophages recruited from the circulation by 28 days post stroke (Besselmann M et al., 2003) these EGFP positive cells are likely to be local phagocytic microglia.

\section{Summary}

In conclusion, we have demonstrated that a strong, statistically significant correlation exists between the volume of EGFP expression in the ipsilateral hemisphere and the volume of structural damage at both acute and later time-points in MacGreen mice, indicating that the degree of inflammation observed post stroke is related to stroke severity. These observations are in agreement with clinical investigations which report that inflammatory parameters such as leukocyte accumulation and serum C-reactive protein levels correlate with both the severity of brain damage and neurological impairment observed post stroke. We also observed changes in the morphology of EGFP positive cells indicating spatial and temporal differences in $\mathrm{M} / \mathrm{M}$ function and phenotype. While it was not possible to trace the origin of the EGFP inflammatory cells in this study, the MacGreen model avoids the limitations of bone-marrow chimeric lines where the effects of irradiation on microglial physiology confound their use in experimental stroke studies. The observations from the current study support the use of MacGreen mice as a clinically relevant model in which to investigate inflammation following stroke. Alterations in EGFP/thionin ratio in MacGreen mice may also be a useful tool assess the efficacy of therapeutic agents targeting inflammation in the sub-acute phase following stroke.

\section{References}

Jin R, Yang G, Li G (2010). Inflammatory mechanisms in ischemic stroke: role of inflammatory cells. J Leukoc Biol 87: $779-789$.

Kriz J. (2006). Inflammation in ischemic brain injury: timing is important. Crit Rev Neurobiol 18: $145-157$.

Nathan C (2002). Points of control in inflammation. Nature 420: 846-852.

Stahel PF, Shohami E, Younis FM, et al. (2000). Experimental closed head injury: analysis of neurological outcome, blood-brain barrier dysfunction, intracranial neutrophil infiltration, and neuronal cell death in mice deficient in genes for pro-inflammatory cytokines. Journal of cerebral blood flow and metabolism: official journal of the International Society of Cerebral Blood Flow and Metabolism 20: 369-380.

Scherbel U, Raghupathi R, Nakamura M, et al. (1999). Differential acute and chronic responses of tumor necrosis factor-deficient mice to experimental brain injury. Proceedings of the National Academy of Sciences of the United States of America 96: 8721-8726.

Kriz J, Lalancette-Hebert M (2009). Inflammation, plasticity and real-time imaging after cerebral ischemia. Acta Neuropathol. May;117: 497-509.

ladecola C, Anrather J (2011). The immunology of stroke: from mechanisms to translation. Nat Med 17: $796-808$. 
Stevens SL, Bao J, Hollis J, Lessov NS, Clark WM, Stenzel-Poore MP (2002). The use of flow cytometry to evaluate temporal changes in inflammatory cells following focal cerebral ischemia in mice. Brain Res 932: 110-119.

Matsumoto $\mathrm{H}$, Kumon Y, Watanabe H, et al. (2008). Accumulation of macrophage-like cells expressing NG2 proteoglycan and lba1 in ischemic core of rat brain after transient middle cerebral artery occlusion. Journal of cerebral blood flow and metabolism: official journal of the International Society of Cerebral Blood Flow and Metabolism 28: 149-163.

Ormstad H, Aass HC, Lund-Sorensen N, Amthor KF, Sandvik L (2011). Serum levels of cytokines and C-reactive protein in acute ischemic stroke patients, and their relationship to stroke lateralization, type, and infarct volume. J Neurol 258: 677-685.

Gelderblom M, Leypoldt F, Steinbach K, et al. (2009). Temporal and spatial dynamics of cerebral immune cell accumulation in stroke. Stroke 40: 1849-1857.

Schilling M, Besselmann M, Leonhard C, Mueller M, Ringelstein EB, Kiefer R (2003). Microglial activation precedes and predominates over macrophage infiltration in transient focal cerebral ischemia: a study in green fluorescent protein transgenic bone marrow chimeric mice. Exp Neurol 183: 25-33.

Schilling M, Besselmann M, Muller M, Strecker JK, Ringelstein EB, Kiefer R (2005). Predominant phagocytic activity of resident microglia over hematogenous macrophages following transient focal cerebral ischemia: an investigation using green fluorescent protein transgenic bone marrow chimeric mice. Exp Neurol 196: 290-297.

Schilling M, Strecker JK, Schabitz WR, Ringelstein EB (2009). Kiefer R. Effects of monocyte chemoattractant protein 1 on blood-borne cell recruitment after transient focal cerebral ischemia in mice. Neuroscience 161: 806-812.

Schilling M, Strecker JK, Ringelstein EB, Schabitz WR, Kiefer R (2009). The role of CC chemokine receptor 2 on microglia activation and blood-borne cell recruitment after transient focal cerebral ischemia in mice. Brain Res 1289: 79-84.

Tanaka R, Komine-Kobayashi M, Mochizuki H, et al. (2003). Migration of enhanced green fluorescent protein expressing bone marrowderived microglia/macrophage into the mouse brain following permanent focal ischemia. Neuroscience 117: 531-539.

Li Y-Q, Chen P, Jain V, Reilly RM, Wong CS (2004). Early radiation-induced endothelial cell loss and blood-spinal cord barrier breakdown in the rat spinal cord. Radiation research 161: 143-152.

Monje ML, Mizumatsu S, Fike JR, Palmer TD (2002). Irradiation induces neural precursor-cell dysfunction. Nature medicine 8: $955-962$.

Davoust N, Vuaillat C, Androdias G, Nataf S (2008). From bone marrow to microglia: barriers and avenues. Trends in immunology 29: 227-234.

Mildner A, Schmidt H, Nitsche M, et al. (2007). Microglia in the adult brain arise from Ly-6ChiCCR2+ monocytes only under defined host conditions. Nature neuroscience 10: 1544-1553.

Wirenfeldt M, Dissing-Olesen L, Babcock AA, et al. (2007). Population control of resident and immigrant microglia by mitosis and apoptosis. The American journal of pathology 17: 617-631.

Rae F, Woods K, Sasmono T, et al. (2007). Characterisation and trophic functions of murine embryonic macrophages based upon the use of a Csf1r-EGFP transgene reporter. Dev Biol 308: 232-246.

Sasmono RT, Oceandy D, Pollard JW, et al. (2003). A macrophage colony-stimulating factor receptor-green fluorescent protein transgene is expressed throughout the mononuclear phagocyte system of the mouse. Blood 101: 1155-1163.

Sasmono RT, Ehrnsperger A, Cronau SL, et al. (2007). Mouse neutrophilic granulocytes express mRNA encoding the macrophage colonystimulating factor receptor (CSF-1R) as well as many other macrophage-specific transcripts and can transdifferentiate into macrophages in vitro in response to CSF-1. J Leukoc Biol 82: 111-123.

Kerr LE, McGregor AL, Amet LE, et al. (2004). Mice overexpressing human caspase 3 appear phenotypically normal but exhibit increased apoptosis and larger lesion volumes in response to transient focal cerebral ischaemia. Cell death and differentiation. 11: 1102-1111.

Hunter AJ, Hatcher J, Virley D, et al. (2000). Functional assessments in mice and rats after focal stroke. Neuropharmacology 39: 806-816.

Gordon RJ, Tattersfield AS, Vazey EM, et al. (2007). Temporal profile of subventricular zone progenitor cell migration following quinolinic acid-induced striatal cell loss. Neuroscience 146: 1704-1718.

Maucksch C, McGregor AL, Yang M, Gordon RJ, Connor B (2013). IGF-I redirects doublecortin-positive cell migration in the normal adult rat brain. Neuroscience. 241: 106-115.

Sasmono RT, Williams E (2012). Generation and characterization of MacGreen mice, the Cfs1r-EGFP transgenic mice. Methods Mol Biol 844: 157-176.

Jones CV, Williams TM, Walker KA, et al. (2013). M2 macrophage polarisation is associated with alveolar formation during postnatal lung development. Respiratory research 14: 41.

Hosp JA, Luft AR (2011). Cortical plasticity during motor learning and recovery after ischemic stroke. Neural plasticity $2011: 871296$.

Maeda K, Hata R, Bader M, Walther T, Hossmann KA (1999). Larger anastomoses in angiotensinogen-knockout mice attenuate early metabolic disturbances after middle cerebral artery occlusion. Journal of cerebral blood flow and metabolism: official journal of the International Society of Cerebral Blood Flow and Metabolism 19: 1092-1098.

Maeda K, Hata R, Hossmann KA (1999). Regional metabolic disturbances and cerebrovascular anatomy after permanent middle cerebral artery occlusion in C57black/6 and SV129 mice. Neurobiol Dis 6: 101-108.

Maeda K, Hata R, Hossmann KA 1998 Differences in the cerebrovascular anatomy of C57black/6 and SV129 mice. Neuroreport 9: $1317-1319$. 
Carmichael ST (2005). Rodent models of focal stroke: size, mechanism, and purpose. NeuroRx : the journal of the American Society for Experimental NeuroTherapeutics 2: 396-409.

Goffus AM, Anderson GD, Hoane M (2010). Sustained delivery of nicotinamide limits cortical injury and improves functional recovery following traumatic brain injury. Oxidative medicine and cellular longevity 3: 145-152.

Blaya MO, Bramlett H, Nadoo J, Pieper AA, Dietrich WD (2013) 3rd. Neuroprotective efficacy of a proneurogenic compound after traumatic brain injury. Journal of neurotrauma 31: 476-486

Perego C, Fumagalli S, De Simoni MG (2011). Temporal pattern of expression and colocalization of microglia/macrophage phenotype markers following brain ischemic injury in mice. J Neuroinflammation 8: 174.

Yenari MA, Kauppinen TM, Swanson RA (2010) Microglial activation in stroke: therapeutic targets. Neurotherapeutics 7: 378-391.

Ransohoff RM, Perry VH (2009). Microglial physiology: unique stimuli, specialized responses. Annu Rev Immunol 27: 119-145.

Schroeter M, Jander S, Witte OW, Stoll G (1994). Local immune responses in the rat cerebral cortex after middle cerebral artery occlusion. Journal of neuroimmunology 55: 195-203.

Gomes-Leal W (2012). Microglial physiopathology: how to explain the dual role of microglia after acute neural disorders? Brain and behavior 2: 345-356.

Ritzel RM, Patel AR, Grenier JM, et al. (2015). Functional differences between microglia and monocytes after ischemic stroke. Journal of Neuroinflammation. 12: 106

\section{Acknowledgements}

The authors would like to thank Jacqui Ross and Hilary Holloway (Biomedical Imaging Research Unit, University of Auckland, New Zealand) for assistance and support with microscopy procedures.

\section{Funding}

This project was funded by the New Zealand Pharmacy Education and Research Foundation and the University of Auckland Faculty Research Development Funds. 